\title{
Participatory action research to pilot a model of mental health service user involvement in an Ethiopian rural primary healthcare setting: study protocol
}

Sisay Abayneh ${ }^{1 *}$, Heidi Lempp ${ }^{2}$ and Charlotte Hanlon ${ }^{1,3,4}$

\begin{abstract}
Background: Involvement of service-users at all levels of the mental health system is a policy imperative in many countries internationally. However, putting policy into practice seems complex; little is known about how best to involve service users and efforts are often criticized for being tokenistic. In low-and-middle income countries, less attention has been given to the roles of service users within mental health systems. The proposed study is part of a larger project intended to develop service-user involvement in mental health system strengthening in Ethiopia. A Theory of Change (ToC) model has already been developed through a participatory approach. This study protocol aims to describe the theoretical background and methods to pilot this model using participatory action research (PAR) and explore participants' experience of involvement.
\end{abstract}

Methods: The proposed study will apply a PAR approach situated in critical social theory and conduct a phenomenological case study to find out participants' experience of involvement. This will be conducted in three stages. The focus of Stage 1 will be to(i) establish a Research Advisory Group (RAG), and Research Participant Group (RPG) at district and primary healthcare facility levels, respectively, and (ii) identify and prioritize potential areas of concern for involvement in the domains of advocacy, service planning and development, monitoring and improving service quality. In Stage 2, we will work with the RPG to develop a plan of action for the selected area. Stage 3 will aim to assist the RPG to implement and evaluate the plan of action. Process indicators and observation will be combined with in-depth interviews with participants to elicit their experiences of involvement. Thematic content analysis will be used.

Discussion: The participatory approach to mental health service user involvement in health system strengthening employed by this study will support the implementation of solutions through locally relevant and contextualized actions. Findings from this study will contribute to the body of knowledge towards understanding the complexity of implementation of service user involvement and refine the ToC model for transferability to similar settings.

Keywords: Participatory research, service user involvement, action research, Sub-Saharan Africa, Mental health, Patient and public involvement

\footnotetext{
* Correspondence: abaynehsisay70@gmail.com

${ }^{1}$ College of Health Sciences, School of Medicine, Department of Psychiatry, WHO Collaborating Centre for Mental Health Research and Capacity-Building, Addis Ababa University, Addis Ababa, Ethiopia

Full list of author information is available at the end of the article
}

(c) The Author(s). 2020 Open Access This article is distributed under the terms of the Creative Commons Attribution 4.0 International License (http://creativecommons.org/licenses/by/4.0/), which permits unrestricted use, distribution, and reproduction in any medium, provided you give appropriate credit to the original author(s) and the source, provide a link to the Creative Commons license, and indicate if changes were made. The Creative Commons Public Domain Dedication waiver (http://creativecommons.org/publicdomain/zero/1.0/) applies to the data made available in this article, unless otherwise stated. 


\section{Plain English summary}

In order to improve mental health care, it is vital that service planners, managers and health professionals work closely with service users. Service users are experts by experience. They can help to hold services to account and make sure that services reach the people who need them in a fair way. In developing countries, the voices of mental health service users are doubly important to make sure that care is respectful, appropriate and of good quality. But in most developing countries, service user voices are not heard. The aim of this paper is to describe our plans to try out a model of service users and health professionals working together to improve mental health care. The setting will be primary care services in a rural district of Ethiopia. We will set up two groups. Group 1 is called the 'Research Advisory Group'. The members of this group will be mental health service users, health professionals, officials and community representatives from the district. Group 1 will decide on which problems are most important. Group 2 is called the 'Research Participant Group'. This group includes service users, their caregivers, health professionals and health managers at a primary care facility. Group 2 will work out how to address the top priority problems. They will then put the plan into action. Together the groups will help to improve mental health care. At the end of the study we will understand more about how services users can be at the heart of improving mental health care in a low-resource African country.

\section{Background}

The importance of involvement of service users and their caregivers (hereafter referred to as 'service-users') at all levels of the mental health system has been recognised globally $[1,2]$. The concept of involvement (alternatively referred to as participation or engagement) [3-5] is defined as "a process by which people are enabled to become actively and genuinely involved in defining the issues of concern to them, in making decisions about factors that affect their lives, in formulating and implementing policies, in planning, developing and delivering services and in taking action to achieve change" [6]. There is explicit international policy direction from the World Health Organization for national mental health systems to empower and involve service-users in mental health advocacy, policy, planning, legislation, service provision, monitoring, research and evaluation $[7,8]$. The same directive has become a policy imperative and is therefore firmly embedded in policy documents of many high income countries $[1,9]$.

In low-and-middle income countries (LMICs), where more than $80 \%$ of service-users are living [10], there is less prioritization and government support for either mental health care provision or involvement of service users $[10,11]$. In many of these countries, there are no policies and laws to direct mental health programs and/ or the policies and laws are not aligned with human rights recommendations (e.g., social care, participation) or are poorly implemented $[10,11]$. Service-users are exposed to stigma and discrimination [12, 13] and have several unmet needs [13], exemplified by suffering of illness and disability [14], impoverishment [15], premature mortality [16, 17], and human rights abuses(e.g., being chained or kept in isolation) [12, 18]. Studies suggest that service-user involvement can also protect and promote human rights $[19,20]$. In LMICs, service-user involvement has been widely recommended as an essential ingredient to strengthen weak mental health systems [21, 22], increasing the likelihood of scale-up of appropriate, quality mental healthcare [23, 24] and thereby reducing the treatment gap $[8,25]$. However, little is known about how best to optimize and lasting involvement of service-users [5, 26]. Service-users are often excluded (rendered invisible and voiceless) from their rights to meaningful participation in decisions that have direct impact on their lives $[18,26,27]$; and are at risk of being left behind during efforts to expand universal health coverage [28].

Methods for service-user involvement have been criticized for the lack of a participatory approach/inclusivity, and being unable to move beyond a tokenistic mode of participation $[1,2,26,29]$. One promising approach to address these criticisms is Participatory Action Research (PAR). The PAR approach is highly conducive to enable marginalized people (in this case people with lived experience of mental illness) to be meaningfully involved in areas of concern to them through developing their capacities and address more holistically the complex factors that hinder their involvement [30-32]. Our recent systematic review (Abayneh et al., in progress) found that PAR is a well-established approach to involve service-users within mental health systems in highincome countries; however, there are few similar studies from LMICs.

\section{Objectives}

The proposed study is informed by a larger project, ongoing since 2014, intended to develop service-user involvement in mental health systems strengthening in Ethiopia. The aim of this paper is to describe the theoretical foundations and methods for a PAR case study of piloting a Theory of Change model for service-user involvement in mental healthcare in a primary health care setting in rural Ethiopia. The specific objectives are to:

- Identify, prioritise and select an area of concern with respect to the integration of mental health into primary healthcare as a focus for involvement of 
service-users, from the perspectives of service-users, caregivers, health professionals/managers and other key community stakeholders.

- Develop plans for action for the selected area of concern.

- Assist service-users, caregivers, health professionals and managers in the implementation of the plan of action.

- Evaluate the process of, and explore the experience of, involvement of service-users, caregivers, health professionals and managers in the PAR activity.

\section{Theoretical Foundation}

Historically, mental health service-users have been excluded from participation in many mainstream social structures, disqualified as knowers and in knowledge production, because they are construed as irrational, unreasonable, incoherent, lacking in insight, deviant from standards of normalcy, unpredictable, unsafe to themselves and others, victims or deficient of mental capacity [33-35], and considered to have a flawed or spoiled identity [33, 36]. Within the mental health system, knowledge gained through formal education is often more highly valued than the experiential knowledge of service-users gained through lived experiences [33, 37, 38]. Although health professionals and researchers have important perspectives on science and practice, service-users can contribute their unique expertise as individuals with lived experiences of their condition and as a recipient of the healthcare services.

When service-users do have contact with mental health services, the system can hinder [39] or deprive them of any real chance to participate, or their input may be devalued in decision-making with respect to aspects affecting their lives [29, 33, 40]. Health systems value and legitimize service providers to act in the "best interest" of service-users [36, 41]. Service-users may have little control over either the nature of the services they receive or the evidence base that legitimizes these services [33, 37, 42]. They have described the exclusion and neglect of lived experiences from knowledge production as 'false and potentially dangerous views of the world' [43] and have highlighted the crucial contribution that their 'experiential knowledge' has to bring to the 'evidence table' $[38,44,45]$, including constructing alternative narratives of experiences and new forms of knowledge [46, 47]. There are also a range of international studies supporting the desirability of lived experience and knowledge for health systems strengthening $[48,49]$. However, pervasive stigmatizing attitudes and discrimination $[12,50,51]$ at multilevel tend to disqualify service-users from full social acceptance, marginalize them and hinder their active involvement [33, 36, 52].Furthermore, because of these negative attitudes and practices, service users may experience powerlessness, consider themselves as 'lesser citizens' or feel unable to act, worthless and incompetent $[53,54]$, commonly described as "internalized oppression" [55].

Given these factors, the authors argue that serviceuser involvement needs to be approached within a critical paradigm [56, 57]. More specifically, we choose to ground the proposed study in critical social theory (CST) [58] with focus on Habermas's theory of communicative space and action [59].The choice of CST has significance in several ways for the proposed study. First, CST can offer a 'communicative space' required to create fora for service-users and other stakeholders to engage in dialogue to reach inter-subjective agreement, mutual understanding, and consensus to guide deliberate, and collaborative social action [60-62]. Communicative space, as employed in this protocol, refers to the spaces in time and place where service-users, caregivers, health professionals/managers and other key stakeholders come together in the PAR process [62, 63], and within created social arenas (e.g., mutual recognition, trusting relationship, reciprocal perspective taking, a shared willingness to consider one's own conditions, learning from each other, reaching common ground for action, sense of agency) $[64,65]$.

Second, CST guides towards recognizing the social, economic, political, and historical contexts that shape human thought and action, and the social structures that have historically served to oppress certain groups in society(e.g., persons with lived experience of mental health conditions) [66-68]. CST can give clues about how to transform social relations of power and enable service-users through (a) expose injustice (through critical analysis and questioning of longstanding established rules, beliefs and practices and conceptualizations about service-users); (b) challenging relationships of domination that exist within the lives of service-users, and allowing them to engage on an equal footing by bringing service-users, health professionals and health administrators to collaborate on a common issue [60-62], and (c) creating opportunities for service users to gain experiences of emancipatory knowledge and greater awareness about their situation, break attitudes of silence, gain confidence and abilities, open themselves up to new ways of understanding, take effective action to alter unjust conditions and structures [69], to formulate alternative stories that are empowering $[65,70]$, and gain more control over their situation $[68,71,72]$. Third, CST is based on a capability/strength-based approach of participation [69],engages directly with service--users [73] and acknowledges service-user knowledge as valid, encouraging mutual recognition and sharing of perspectives [59, 73]. 


\section{Methods \\ Setting}

The proposed study is part of a larger project that was instigated as part of the 'Emerging mental health systems in low- and middle-income countries'(Emerald) project, which investigated the health system requirements for successful implementation of integrated mental health care in six LMICs(Ethiopia, India, Nepal, Nigeria, South Africa and Uganda) [74, 75]. The study will take place in Sodo district, a rural district located in the Gurage Zone of the Southern Nations, Nationalities and Peoples' region, about $100 \mathrm{~km}$ south of Addis Ababa. The district had a population of 161,952 people in 2007 [76]. Around $90 \%$ of the district population reside in rural areas and are reliant on subsistence farming [77]. The district population is predominantly composed of the Gurage ethnic group and followers of Orthodox Christianity. The official language of the district is Amharic [76]. There are 58 sub-districts or kebeles (the smallest administrative units with 2000 to 5000 people each), which are both geographically and climatically diverse. There is one primary hospital with an outpatient psychiatric service (run by a psychiatric nurse) in the main town and eight health centres, four of which are located within the three towns of the district. The primary hospital and all eight health centres have functioning mental health services using a task-shared model of care. Each health centre serves about five sub-districts, comprising a population of about 25,000-40,000 people [78]. Each sub-district has a health post (lowest statutory healthcare facility). The health posts are staffed by a pair of community health workers called health extension workers (HEWs). The HEWs are high school graduates with one year of training in sixteen packages of care which cover four main areas: disease prevention and control, family health, hygiene and environmental sanitation, and health education and communication [79].A minority of HEWs have received training in mental health as part of their upgrading to level IV.

Sodo district is the research and implementation site for the PRogramme for Improving Mental health carE (PRIME) [80, 81]. As part of PRIME, primary care staff in Sodo district have been trained to deliver packages of care for people with mental health conditions, including prescription of antipsychotic medication, follow-up, limited adherence support, basic psycho-education and community awareness-raising of mental illness [82]. PRIME established a multi-sectoral community health advisory board with representatives from key members of the district leadership (security, gender office, women and youth affairs, religious affairs and education), the community and service users and caregivers, and was chaired by the head of the district health office [82]. The CAB met twice a year to oversee and advise PRIME [83].

\section{Design}

This section describes the research design, rationale and stages of the proposed study. We propose to use a PAR approach [84] and a phenomenological case study [85] to explore participants' experiences of involvement. In this study, drawing on work by Rouleau et al. [86], and Nelson et al. [87], PAR is defined as the: (i) valuation, mobilization and legitimization of service user experiential knowledge of living with a particular health conditio$\mathrm{n}$ (e.g., mental illness); (ii) conduct of research that focuses on service users' concerns, participation, and outcomes; and (iii) active partnership among a variety of stakeholders/actors(e.g., researchers, health professionals, decision makers, organizations, service-users) [86], for the purpose of taking action and making change [87]. PAR is the approach of choice for the proposed study for several reasons.

First, PAR has a collection of research methods (epistemological pluralism) [88] that is uniquely suitable to address complex problems (such as service-user involvement $[3,89]$ ), build evidence in areas that lack an empirical evidence base and find practical solutions in the areas of health systems strengthening, implementation research, various health and social care settings [88, 90, 91]. PAR has been increasingly said to be more robust than other approaches because the process (a) simultaneously generates knowledge and initiates actions informed by that knowledge [73, 92, 93], (b) makes knowledge accessible and relevant to stakeholders to underpin change [93, 94], (c) relies on a commitment to bring together theoretical and methodological expertise and the practical knowledge of non-academic participants ('creates self-critical communities' $[95,96])$, (d) shares leadership and resources to address issues in specific systems [67, 97], and (e) enables co-design of culturally appropriate and effective interventions, their implementation and collaborative evaluation of impact [60,95].

Second, although many programme theories articulate intended changes [98], engage with the complexity of interventions and provide a framework to guide action, monitoring and evaluation [99], there is little empirical evidence of how theories can be applied in practice [100-102]. Hence, there have been calls for PAR in theory-based implementation and evaluations [103-105]. Early integration of Theory of Change (ToC) and PAR during planning and implementation is recommended $[103,106,107]$. To the best of our knowledge, no one has yet offered a description of how to combine ToC and PAR to apply service-user involvement in mental health systems. We attempted to address this gap by taking cues from the evidence base on combining ToC and PAR from other disciplines (e.g., agriculture [100, 108], education [102], development studies [109], programme evaluation $[110,111]$ and implementation sciences [112]. 
For our proposed study, combining ToC along with PAR is a promising approach for several reasons:

A) At the core of both ToC and PAR is a concern with how and why change takes place. Both anticipate a range of positive changes/outcomes [96, 100] including: individual level outcomes(e.g., advancing participants' personal and collective sense of agency, social networks) and community level outcomes(awareness raising, stigma reduction, and strengthening community capacity, collaboration) $[54,56,113]$. ToC provides a strong heuristic device for deeper understanding of the implementation context $[100,112,114]$, guides the direction of change and how to achieve the intended transformation, defines collaborative outcomes and surfaces the various layers of interventions with underlying assumptions, and ensures that different perspectives of participants are reflected in the design $[111,115,116]$. However, that alone may not be sufficient to support the actions required to achieve implementation [102, 107, 108]. This gap can be balanced when ToC is combined with PAR, because the cyclical nature of PAR (iterative cycles of reflection, planning and acting), facilitates learning about what, how and why change is unfolding [103, 108, 111].

B) In practice, $\mathrm{ToC}$ and other programme theories may have a problem of reach, i.e., they do not explicitly consider issues of inclusivity and there is little acknowledgement of the way in which power operates to affect the building of collaborative capacity or how this notion needs to be addressed to enable stakeholder participation [106, 107, 117].Without such explicit consideration of power dynamics, ToC approaches may inadvertently reinforce a hierarchical relationship between stakeholders and privilege the perspectives of those in power (e.g., policy makers, professionals) and downplay or even disregard entirely the views of others(e.g., service-users) [116, 118, 119].

These drawbacks may be minimized through the emancipatory and critical theoretical foundation of PAR that seeks to explicitly and intentionally work with a range of stakeholders, including those historically oppressed, disempowered, vulnerable and marginalized groups (e.g., service-users) [62, 107, 112]. The participatory commitment of PAR provides space for diverse forms of expertise and promotes understanding of the different life-worlds of participants [32, 87, 120]. Working in a collaborative and non-hierarchical manner may facilitate deeper understanding of how implementation can be achieved [56, 84, 100, 112, 121]. Furthermore, the dialogue and critical reflection incorporated within PAR enables participants to challenge the status quo of professional-dominated health systems, dismantle unequal power relations between service users/caregivers and those within health systems and society and create fruitful communication [121-123].

C) PAR also rejects objectivist assumptions that distance the researcher and the participants from one another $[121,123]$. The close proximity of working can promote inclusion and confront engrained stigma and prejudices [32, 120, 124]. In line with the contact hypothesis [125] and social contact theory $[125,126]$, positive contact between service-users, health professionals and others within the health system, e.g. by giving them equal status in pursuing common goals, can foster mutual understanding and reduce stigmatizing attitudes [127, 128].

\section{Sessions and stages of the proposed study}

The study procedure will take place in three stages, with cyclical recurring activities involving planning, acting, observing and reflecting, informed by the model proposed by Kemmis and McTaggart [129] (See Table 2 for proposed sessions plans, stages and activities). The three stages are: i) Establishing of groups, identification and prioritization of thematic concerns, ii) Planning of action, and iii) implementation (See Fig. 1, for summary of stages). We anticipate that the participants will need to meet for at least seven weekly sessions for two to three hours. The first two sessions will take place at district level at Buie town, the capital of Sodo district, and the other five sessions within the primary health care facility and will be conducted in Amharic language.

\section{STAGE 1: establishment of groups, discussion of foundational studies, identification and prioritization of concerns}

Formation of cross-stakeholder groups (planning)

For the proposed study, we aim to maximize participation of diverse representatives of the local community through establishing two multi-stakeholder groups that will collaborate and be involved within the research process: (i) a Research Advisory Group (RAG), and (ii) a Research Participant Group (RPG).

\section{Research Advisory Group}

The importance of involving a Research Advisory Group (RAG) was recognized early during the larger study. Although our plan was to establish a new RAG, after our discussion with Sodo district health officials, we agreed to work with the existing community advisory 




Fig. 1 Formative works and stages of the Proposed Study

board $(\mathrm{CAB})$ that had been established to oversee efforts to expand access to mental health care in the district (working with PRIME) [82]. As part of the larger study, the Sodo district $\mathrm{CAB}$ has participated in the coproduction of the ToC for mental health service-user involvement and contributed to a community stakeholder consultative meeting. To ensure a feasible working group size, for the RAG we will purposively select 20 participants from the larger $C A B$. Decisions on who to select will be made collectively with district officials involved in the mental health care programme, based on pre-specified criteria (See Additional file 1for inclusion criteria). In addition we will ensure gender representativeness of participants.

The RAG will play several roles in the proposed study, including: (i) oversee and advise on priority problems for improving mental health care from their local community perspectives, (ii) provide a conduit between the
Research Participant Group(RPG) and the community to ensure that the research findings are put into action and disseminated in their local context; (iii) create a strategy to enable an empowering environment(e.g., through resource mobilization) for service user involvement,(iv) facilitate further consultation and community involvement for service user mobilization and empowerment, and $(\mathrm{v})$ advocate for the protections of rights of service users. The RAG will meet three times during the course of the research in Sodo district: two half-day meetings during Stage 1 and once in Stage 3.

\section{Research Participant Group}

A Research Participant Group (RPG), comprising of up to 12 participants (mental health service-users- $n=4$, caregivers- $n=4$, and health professionals and health facility managers- $n=4$ ), will be convened at a health facility in Sodo district (See Additional file 1for inclusion 
criteria to guide purposive selection of participants). As our research objective is not specific to certain mental health conditions, and to increase the social validation of the study objectives, procedures and outcomes [130], we will included service users with psychosis, depression, epilepsy, and alcohol use disorder. We also try to balance gender representation of participants. The RPG will participate throughout the research process in (i) identification and prioritization of priority problem areas, (ii) identification of specific areas of concern at the health facility level; development, implementation and evaluation of an action plan, and (iii) validation of the research process and local dissemination of the findings of the study. The principal investigator and a research assistant will act as facilitators of the process of prioritization, design, conduct and dissemination of activities of the research study with a view to empowering the RPG at each stage in the PAR cycles.

\section{Discussion of foundational studies, identification and prioritization of potential areas of concern(action)}

This session will include two sets of activities including [1] presentations and discussion about foundational studies, and [2] identification and prioritization of potential areas of concerns as briefly detailed below.

\section{Presentation and discussion about formative works}

The development of this protocol was informed by formative work: (i) a qualitative study, (ii) development of a Theory of Change model for service-user involvement, (iii) capacity building training, (iv) a systematic review, and (v) a community stakeholder consultative meeting (See Fig. 1) in a larger project intended to develop service-user involvement in mental health system strengthening in Ethiopia. In Stage 1 of the proposed study, based on the findings of the formative works, a half-day consultative workshop with representatives from stakeholder groups will be conducted to ascertain the situation of service user involvement in mental health system strengthening in the study site in relation to the global situation. The intention of this protocol is not to report the details of the foundational studies; rather we present a brief overview of the $\mathrm{ToC}$ to inform readers about how that informed the development of this protocol.

\section{Theory of Change}

As part of the larger study, a generic ToC for serviceuser and caregiver involvement in mental health system strengthening in Ethiopia was co-produced with stakeholder groups, including service users, caregivers, psychiatrists, researchers, and statutory and non-statutory community representatives. The ToC helped to make explicit the hypothesized pathways to achieve the long- term outcome (derived by consensus) of "improved physical and mental health, economic productivity and social inclusion for service users, and improved life satisfaction, including economic capacity for caregivers". The $\mathrm{ToC}$ also allowed identification of necessary preconditions for success, programme levels for an intervention (service user/caregivers, health facility and community), indicators of success, assumptions underpinning the pathway and the types of interventions needed. In the ToC, capacity building training for service users, caregivers, and health professionals/managers, PAR with stakeholder groups, inter-sectoral collaboration, and service-user mobilization were identified as programme interventions to enable service user involvement to achieve the long-term outcome.

The co-production of the ToC with diverse stakeholder groups and the embedded PAR in both the design and implementation of the interventions will enable the $\mathrm{ToC}$ to be responsive to local needs [131-133]. However, in the same way that most ToCs are comprehensive road maps for the implementation of a programme [102, 103, 107], our one was also generic and cannot show the specific target of action for service user and caregiver involvement. Service users can potentially be involved in each domain of the mental health system (service planning, service development and delivery, service quality improvement, education/training, service promotion and advocacy); however, there is no evidence-based algorithm to determine how to prioritize the domains. The embedded PAR as an intervention component in our ToC can help to identify and prioritize problems, the specific targets of action as well as the domains of mental health systems, and develop a plan of action based on unique local contexts and strengths by involving service users and other key stakeholders $[102,107,134]$. Therefore, to specify the ToC interventions fora primary healthcare setting, we will conduct a half-day participatory interactive workshop involving stakeholder groups (See prioritization section).

\section{Identification and Prioritization of Thematic Concerns (Action, Observation and Reflection)}

In the proposed study, we will use PAR to initiate a oneday priority setting exercise by bringing together service users, caregivers, health professionals/managers, and CAB members (including RPG and RAG) to generate a list of their top priority problems for research in involving service users in mental health system strengthening in Sodo district. The study will be informed by guidance from the James Lind Alliance (JLA) [135] to ensure a balanced, inclusive and transparent process for priority problems identification, and the Nominal Group Technique (NGT) to establish consensus, prioritize and rank the thematic concerns [136-138]. The JLA approach enables us to create an environment that encourages open 
discussion, respect for diversity and clarity of thought, and also has been used to identify research priorities in several areas including mental health $[135,139]$.

For the proposed study, potential priority problems for involvement of service users in mental health systems improvement will be identified and short listed by stakeholder groups in a 1-day workshop in Sodo district using the five step JLA process [135](see Table 1 for details of the steps). Given the complex nature of service user involvement within the mental health system [3, 5, 89], and low levels of experience of service users, caregivers and other community stakeholders working together within mental health systems in Ethiopia [140], the principal investigator will provide a list of potential priority problems (questions) extracted from the foundational studies and evidence review (See Additional file 2) to prompt discussion and enable participants to choose problems of relevance to their local context. To reduce the possibility of bias and influence by the potential priority problems/questions, the principal investigator and facilitators will encourage participants to reflect upon the sample priority areas, to modify or drop the potential thematic concerns provided and add their own thematic areas that are most important for themselves.

Although JLA enables the identification of potential priority problems of interest to stakeholders; there is a need to move beyond a focus on uncertainties to the generation of shared priorities, ranking and achieve consensus on the priorities. For this, group decision making processes such as NGT are helpful, because of a wellestablished, multistep facilitated group interactive process through increased engagement of relevant stakeholder$\mathrm{s}$ (including those otherwise excluded groups)on concerns that are important and matter to them [136, 137]. The processes of silent generation of responses, round-robin listening and independent voting ensures the participation of all individuals. The structure of voting and discussion allows the person to express a view, influence decisions, avoid conformity or social pressure, and individual judgments can be aggregated into group conclusions whereby anonymous individual rank-orderings are aggregated across members to determine the relative importance of all responses $[136,137]$. For the proposed study, we integrated NGT with stage 5 in JLA which seems ideally suited for PAR and consistent with the critical social theory, NGT will give all participants a voice, and produce priorities and practical change $[137,138]$. Following the NGT, the participants will generate, revise, vote on and rank priority problems of importance to their local context (See Table 1 for details). Participants will rate the importance of the thematic concerns on a 5-point Likert scale (very high priority to very low priority).The priority list that is created will be grouped into broad thematic areas of domains of mental health systems (e.g., research, quality improvement, advocacy) by consensus and using an inductive approach.

\section{STAGE 2. Plan of action development}

In stage 2, the key themes generated and prioritized from Stage 1 will be presented back to the RPG and reviewed at a healthcare facility in Sodo district. The RPG will discuss the prioritized areas of concern and choose one concrete theme/problem to be addressed in their specific health facility/setting, identify lists of strategies to solve the problem and develop a plan of action for that specific health facility/local setting. During this stage, the cycles of PAR (including planning, acting, observing and reflecting) will be undertaken (See Table 2).

\section{Stage 3: implementation and evaluation}

The focus of this stage is actual field implementation of the proposed strategies and action priorities in Stage2, and evaluation of the process. In partnership with the RPG, assessment of the local context of the health facility will be conducted by the principal investigator, including identification of potential opportunities and barriers to implementing the agreed actions. The entire implementation process will be underpinned by the cyclical PAR activities of planning, acting, observing and reflection (See Table 2). However, as this study is also of interest for academic purposes (principal investigator), the authors anticipate time pressure may hinder the full involvement of the principal investigator in the final cycles. Hence, some key strategies and actions that can be implemented within the time frame of the study and will be identified during the initial discussions with RPG, implemented and evaluated(See Table 2) as a proof of concept. As the action stages evolve, the RPG will be empowered to become autonomous to take actions and effectively implement their action plan in their areas of priority, and the role of the principal investigator will become advisory and consultative [141].

\section{Data collection and analysis}

Multiple sources and methods will be used to collect data. All participants (RAG and RPG) will fill out socio-demographic questionnaires at the beginning of the first session. The following types of data will be collected: meeting minutes, written documentation of prioritization and consensus processes, reflective field notes(reflective journal) of obstacles and successes of the research process, participant observation during all group discussions, and anonymous feedback from the participants about the process, and audio recordings of all sessions. After the last session of PAR, indepth interviews will be conducted with the RPG members to explore their experiences of involvement in the PAR processes. 
Table 1 Procedures to thematic concern identification and prioritization

Stages
Step 1. Establishing the priority setting
Partnership and defining scope

Partnership and defining scope

Step 2. Gathering and identifying questions

Step 3. Reducing the questions and processing uncertainties

Step 4. Interim Prioritization

Step 5. Final Priority setting

\section{Description}

A cross-stakeholder groups $(n=20-25)$ including RPG and RAG will be selected with maximum variation comprising of relevant statutory and non-statutory organization representatives and individuals that can reach and advocate for, mobilizing resource, empower and support service users for involvement in mental health systems strengthening. The stakeholder groups will be invited to a half-day consultative meeting and discuss on the findings of foundational studies in Phases 1\&2 about service user involvement so as to raise awareness, create the need for collaboration, and define the scope of the study for future action.

The cross-stakeholder groups will be invited in a 1-day thematic concern identification and prioritization exercise at Sodo district.

The participants will be divided into four homogeneous groups (service user, caregivers, and health professionals, community stakeholders) so that the participants are comfortable voicing their opinions.

Each group separately will be asked to list as many priority questions from their own perspectives perceived as the most important challenges to be addressed for service user and caregiver involvement in mental health system improvement.

Facilitators will gather the list of questions/thematic concerns in each group and record in a flip chart

In addition, each group will be provided with the pre-generated lists of potential priority areas (Additional file 2) to discuss on, augment their priorities, and identify additional priorities

Each group will present their list of thematic concerns in a plenary session.

Facilitators will create a list of unique themes by merging duplicates and overlapping questions (issues) on a flip chart.

The identified themes will be grouped into key themes with list of specific concerns/issues.

The consolidated lists of priorities will be distributed to the homogenous groups to identify their top 10 research priorities in the order of perceived importance that they think need be the focus of research involving service users within Sodo district using pre-set criteria (e.g., relevance local primary health care and community, public health significance, magnitude of the problem, severity, feasibility/amenability to change with local context). Accordingly, each participant will select his/her top ten priorities and ranks them by giving each priority a score between 1 (lowest) and 10(highest). The top list of each participant within the homogeneous groups will be combined by consensus and presented in a plenary session for listing the 10 priorities considered most important by all stakeholders group and reach consensus.

The participants will be organized into nominal groups, and generate their top five priorities and rank them in orders of importance. This will follow the following five steps.

a. The participants will be divided into four groups with balance of service user, caregivers, health professionals and community stakeholders and each participant within each group will be asked to silently generate top five priorities from the top 10 lists generated.

b. A round robin approach of recording of priorities will be used to collate priorities, that is, each participant in turn will be asked to read one priority off the list within each group. This priority will be written on flip chart by a facilitator of each group.

c. Once all the priorities are written on the flip chart an open discussion will be conducted to allow all participants within each group to discuss, clarify, dispute and discarded or add or modify a priority within their groups.

d. The final lists of priorities from each group will be presented in a plenary session, bring similar priorities together on a flip chart, and will be discussed with the whole group in order to ensure that all participants understand and approve of the congregated priorities. e. Finally, each participants will be provided with the combined consensus priorities and asked individually and anonymously, to rank all the five most important priorities in the order of importance by giving five to the highest valued priority, the next most important, a value of four and so on progressively down to the least important which will be assigned a value of 1. A mean priority score for each priority across all groups will be calculated by summarising ranking scores and dividing this by the maximum possible ranking score of that priority. The maximum possible ranking score for a given priority will be calculated by multiplying the number of participants who considered the priority by 5 (the maximum rank) Similar NGT will be conducted with RPG at a health facility level to identify and establish two top priorities for action trial.
Thematic analysis of the data will be conducted [142]. The data analysis method will be based on Interpretative Phenomenological approach, which places the participants' experiences at the core [143]. We expect that it will be challenging to involve service user in the data analysis, in the true sense of the word, hence the principal investigator will lead the data analysis, and the results of the analysis will be fed back to the participants for member checking. 
Table 2 Summary of stages, activities, and session plans for the proposed study

\begin{tabular}{|c|c|c|c|}
\hline Stages & Cyclical activities & Descriptions & Sessions \\
\hline \multirow[t]{4}{*}{$\begin{array}{l}\text { Stage 1: Formation of } \\
\text { stakeholders Groups, and } \\
\text { Consultative Workshop }\end{array}$} & Planning & $\begin{array}{l}\text { Identify and establish cross-stakeholder groups that services as a reference } \\
\text { group, and working group together with Sodo district health office } \\
\text { Getting stakeholder groups and agree on time and place for regular sessions } \\
\text { Develop summary of findings from foundational studies } \\
\text { Identify and prioritize top thematic concerns }\end{array}$ & 1 \\
\hline & Action & $\begin{array}{l}\text { Present and discuss on foundational studies in a consultative workshop with } \\
\text { stakeholder groups } \\
\text { Systematically identify thematic concerns through small homogenous groups } \\
\text { and heterogeneous group discussions, prioritize thematic concerns using } \\
\text { Nominal Group Techniques }\end{array}$ & 2 \\
\hline & Observing & $\begin{array}{l}\text { Collect key thematic concerns and priorities generated in small group and } \\
\text { plenary sessions, through audio-recording, capture minutes, field notes } \\
\text { A research assistant will record field notes on group dynamics and } \\
\text { interactions and on the context surrounding the discussion. }\end{array}$ & $1-2$ \\
\hline & Reflection & $\begin{array}{l}\text { Discuss on and reach consensus on priority areas } \\
\text { Reflect within homogeneous groups, heterogeneous; compare the reports of } \\
\text { each group } \\
\text { The stakeholder groups make sense of what has happed through thinking } \\
\text { about how it fits with their experiences and local contexts using criteria }\end{array}$ & 2 \\
\hline \multirow[t]{4}{*}{ Stage2: Planning of action } & Planning & $\begin{array}{l}\text { Reach common understanding between RPG and the researchers and } \\
\text { assistants what the research involves and ensure consent to participate } \\
\text { RPG agree on time, place, number of sessions per week and duration of the } \\
\text { sessions at primary health facility level } \\
\text { Review the thematic priorities identified in Stage 1, discuss, select and } \\
\text { prioritize two thematic concerns for action as trial of proof of concepts } \\
\text { Generate set of solutions and design intervention strategies }\end{array}$ & 3 \\
\hline & Action & $\begin{array}{l}\text { Work with RPG and develop viable and realistic change strategies taking into } \\
\text { account their local realities; set evaluation strategies for actions }\end{array}$ & 4 \\
\hline & Observing & $\begin{array}{l}\text { Observe and document the process through notes, and audio-recordings } \\
\text { Evaluate participation and representation }\end{array}$ & $3-4$ \\
\hline & Reflection & $\begin{array}{l}\text { Continuous reflection throughout the action planning phases on data from } \\
\text { observation, field notes and reflect on the action options } \\
\text { Examine whether the proposed improvement methods is feasible in terms, } \\
\text { time, additional resources availability, and local experiences }\end{array}$ & $3-4$ \\
\hline \multirow[t]{4}{*}{$\begin{array}{l}\text { Stage3: Implementation and } \\
\text { evaluation }\end{array}$} & Planning & $\begin{array}{l}\text { Review of the plan action with RPG and reach agreement about the way } \\
\text { strategies would be put into operation and how to document observations } \\
\text { Designing implementation strategies and action } \\
\text { Discuss about and set implementation indicators } \\
\text { Discuss and research consensus how the RPG will continue with the PAR } \\
\text { processes on own }\end{array}$ & 5 \\
\hline & Action & $\begin{array}{l}\text { Implementation meeting with RPG } \\
\text { Reach agreement about the way the program would be put into operation } \\
\text { and how to document observations } \\
\text { Select few interventions and commence as trial of proof of concept } \\
\text { Discuss and research consensus how the RPG will continue with the PAR } \\
\text { processes on their own }\end{array}$ & 6 \\
\hline & Observing & $\begin{array}{l}\text { Document the trial process through taking detailed field notes, observation } \\
\text { and discussion with RPG } \\
\text { Preliminary analysis and findings of the process will be collected } \\
\text { Conducted in-depth interviews with RPG to ascertain their perceptions and } \\
\text { experiences of the process of PAR }\end{array}$ & 6 \\
\hline & Reflection & $\begin{array}{l}\text { Conduct evaluation meeting with RPG and collect feedback about the } \\
\text { process of the PAR process, and reflect on the process of implementation } \\
\text { Identify options for further PAR and action with or without academic } \\
\text { researchers }\end{array}$ & 7 \\
\hline
\end{tabular}

\section{Rigour}

Several measures will be employed to increase the rigour, authenticity, and trustworthiness of the proposed data collection and analysis. Bias in data collection and coding by the principal investigator and research assistant will be reduced through regular discussions(to maintain reflexivity)with the RPG [144, 145]. In addition, the principal investigator will acknowledge and record 
sources of potential personal bias that may influence the process of data collection and analysis as a result of existing networks and connections. The process will provide an audit trail of the reflective process; compliance with the criteria of confirmability data will be ensured by audio recording of discussion groups [144, 145]. Increased credibility will be achieved through prolonged engagement of the principal investigator within the setting, triangulation of multiple data sources and methods(e.g., written minutes, observations, field notes, and in-depth interviews), and regular member checking of raw data, and reports, which will support sustained dialogue with participants, and development of authentic, trusting rapport between the researcher and participants $[144,145]$. Transferability of the study will be increased through sufficient and rich contextual description of the study setting, thick contextual data and activities details, and proper data documentation to allow others to analyze the situation and research outcomes based on setting and context [145].

\section{Strengths and limitations}

The use of PAR to pilot the model for service user involvement within the health systems is a new experience, and to our knowledge, there have not been any studies that have piloted and evaluated $\mathrm{ToC}$ in conjunction with PAR for service users and caregiver involvement in mental health systems strengthening in Ethiopia or other LMICs. The PAR approach will enable us to improve the model to fit the needs of service users and improve its relevance; the co-design of the model also ensures its local applicability and sufficient adaptability to be transferable to other health facilities in LMICs. The use of PAR that embedded within critical social theory provides a strong theoretical foundation, which bring stakeholders together to define for themselves their needs and experiences, identify any areas of concern, develop a plan of action, and support the implementation of solutions. The findings of the study are likely to result in an increased understanding of complex phenomena of service user involvement; can contribute in refining the $\mathrm{ToC}$ model for better transferability, and may provide future researchers with useful insights and foresights in the development and implementation of more stakeholder inclusive initiatives for service user involvement in mental health systems strengthening in Ethiopia and other similar LMICs.

There are several limitations to the proposed study. The proposed pilot study is a small-scale exploratory study and there is no comparison group. A comparative study of larger scale of the proposed study is justified for interested researchers for more rigorous evaluation to provide further support to the impact of the implementation of service user/caregiver involvement experiences.
The selection of the participants and pilot site is purposive and so this limits the transferability of the study findings. However, the aim of our pilot study was not to be representative of the whole landscape of primary care clinics and service-users, but to co-design a model with service-user, caregivers, primary health care professionals and health facility managers. The low literacy levels of service users and caregivers may be a barrier to involvement in all stages of the PAR process.

\section{Supplementary information}

Supplementary information accompanies this paper at https://doi.org/10 1186/s40900-019-0175-x.

Additional file 1. Inclusion criteria for cross-stakeholder participants in the proposed study.

Additional file 2. Potential priority concerns to strengthen mental health systems involving service users.

\section{Abbreviations}

CAB: Community Advisory Board; CST: Critical Social Theory;

Emerald: Emerging mental health systems in low- and middle-income countries; HEWs: Health extension workers; JLA: James Lind Alliance; LMICs: lowand middle-income countries; NGT: Nominal Group Technique;

PAR: Participatory Action Research; PRIME: PRogramme for Improving Mental health carE; RAG: Research Advisory Groups; RPG: Research Participant

Groups; ToC: Theory of Change

\section{Acknowledgements}

Thank you to all of service users, caregivers, CAB members, core research teams, senior psychiatrists, and Sodo district officials who took in the foundational studies. Special thanks to the PRIME field coordinators (Moges, Tigist and Bogale), who assisted us during the foundational studies and captured minutes during the workshops.

\section{Authors' contributions}

SA led and contributed to designing the study protocol with inputs from $\mathrm{CH}$ and $\mathrm{HL}$. All authors were involved in the protocol design, critically reviewed the paper. All authors read and approved the final manuscript.

\section{Funding}

This study has been part funded by a grant from the Psychiatry Research Trust, the Institute of Psychiatry, Psychology and Neuroscience at King's College London, and with additional funding from Addis Ababa University. The views and opinions expressed are those of the authors, and not those of the funding organizations.CH (King's College London and AAU) is funded by the National Institute of Health Research (NIHR) Global Health Research Unit on Health System Strengthening in Sub-Saharan Africa, King's College London (GHRU 16/136/54) using UK aid from the UK Government. The views expressed in this publication are those of the authors and not necessarily those of the NIHR or the Department of Health and Social Care. CH additionally receives support from the Africa Mental Health Research Initiative (AMARI) as part of the DELTAS Africa Initiative (DEL-15-01).

Availability of data and materials Not applicable.

Ethics approval and consent to participate

Ethical approval was obtained from the Scientific Committee of the Department of Psychiatry and the Institutional Review Board of Addis Ababa University College of Health Sciences (005/2016). All participants will be provided with written information about the study and will give written formal consent voluntarily to participate in the study. All stakeholders will be freely able to withdraw at any point, and those who choose not to participate in the study will be informed that they will not be disadvantaged in any way in their future mental health care provided within the Sodo District. 


\section{Consent for publication}

Not applicable.

\section{Competing interests}

The authors declare that they have no competing interests.

\section{Author details}

'College of Health Sciences, School of Medicine, Department of Psychiatry, WHO Collaborating Centre for Mental Health Research and Capacity-Building, Addis Ababa University, Addis Ababa, Ethiopia. ${ }^{2}$ King's College London, Centre for Rheumatic Diseases, School of Immunology and Microbial Sciences, Faculty of Life Sciences and Medicine, Weston Education Centre, 10, Cutcombe Rd, London SE5 9RJ, UK. ${ }^{3}$ King's College London, Centre for Global Mental Health, Institute of Psychiatry, Psychology and Neuroscience, 16 De Crespigny Park, London SE5 8AF, UK. ${ }^{4}$ Centre for Innovative Drug Development and Therapeutic Trials for Africa (CDT-Africa), College of Health Sciences, Addis Ababa University, Addis Ababa, Ethiopia.

\section{Received: 12 September 2019 Accepted: 19 December 2019}

\section{Published online: 08 January 2020}

\section{References}

1. Bee P, Price O, Baker J, Lovell K. Systematic synthesis of barriers and facilitators to service user-led care planning. Br J Psychiatry. 2015;207(2):104-14.

2. Stomski NJ, Morrison P. Participation in mental healthcare: a qualitative meta-synthesis. Int J Ment Heal Syst. 2017:11(1):67.

3. Tambuyzer E, Pieters $G$, Van Audenhove C. Patient involvement in mental health care: one size does not fit all. Health Expect. 2011;17(1):138-50.

4. Gallivan J, Kovacs Burns K, Bellows M, Eigenseher C. The many faces of patient engagement. J Participatory Med. 2012;4:e32.

5. Millar SL, Chambers M, Giles M. Service user involvement in mental health care: an evolutionary concept analysis. Health Expect. 2016;19(2):209-21.

6. World Health Organization. Community participation in local health and sustainable development: Approaches and techniques. 2002.

7. World Health Organization. Mental health action plan 2013-2020. Geneva: World Health Organization; 2013. This document was produced in response to the World Health Assembly Resolutions WHA66 8 and WHA65 4 , provides the necessary framework for improving mental health globally from the government to individual level, and provides a strong rationale for collaborative care. 2013.

8. Saxena S, Funk M, Chisholm D. World health assembly adopts comprehensive mental health action plan 2013-2020. Lancet. 2013; 381(9882):1970-1.

9. Lloyd M. Participation in practice a review of service user involvement in mental health nursing. Mental Health Learn Disabil Res Pract. 2010;7(2):195-206.

10. Rathod S, Pinninti N, Irfan M, Gorczynski P, Rathod P, Gega L, et al. Mental health service provision in low-and middle-income countries. Health Serv Insights. 2017;10.

11. Alloh FT, Regmi P, Onche I, Van Teijlingen E, Trenoweth S. Mental health in low-and middle income countries (LMICS): going beyond the need for funding. Health Prospect J Public Health. 2018;17(1):12-7.

12. Semrau M, Evans-Lacko S, Koschorke M, Ashenafi L, Thornicroft G. Stigma and discrimination related to mental illness in low-and middle-income countries. Epidemiol Psychiatr Sci. 2015;24(5):382-94.

13. Heim E, Kohrt B, Koschorke M, Milenova M, Thronicroft G. Reducing menta health-related stigma in primary health care settings in low-and middleincome countries: a systematic review. Epidemiol Psychiatr Sci. 2018:1-10.

14. Whiteford HA, Degenhardt L, Rehm J, Baxter AJ, Ferrari AJ, Erskine HE, et al. Global burden of disease attributable to mental and substance use disorders: findings from the global burden of disease study 2010. Lancet. 2013;382(9904):1575-86.

15. Lund C, Breen A, Flisher AJ, Kakuma R, Corrigall J, Joska JA, et al. Poverty and common mental disorders in low and middle income countries: a systematic review. Soc Sci Med. 2010;71(3):517-28.

16. Fekadu A, Medhin G, Kebede D, Alem A, Cleare AJ, Prince M, et al. Excess mortality in severe mental illness: 10-year population-based cohort study in rural Ethiopia. Br J Psychiatry. 2015;206(4):289-96.

17. Liu NH, Daumit GL, Dua T, Aquila R, Charlson F, Cuijpers P, et al. Excess mortality in persons with severe mental disorders: a multilevel intervention framework and priorities for clinical practice, policy and research agendas. World Psychiatry. 2017;16(1):30-40.
18. Drew N, Funk M, Tang S, Lamichhane J, Chávez E, Katontoka S, et al. Human rights violations of people with mental and psychosocial disabilities: an unresolved global crisis. Lancet. 2011;378(9803):1664-75.

19. Thornicroft G, Alem A, Dos Santos RA, Barley E, Drake RE, Gregorio G, et al. WPA guidance on steps, obstacles and mistakes to avoid in the implementation of community mental health care. World Psychiatry. 2010; 9(2):67-77.

20. Raja S, Underhill C, Shrestha P, Sunder U, Mannarath S, Wood SK, et al. Integrating mental health and development: a case study of the BasicNeeds model in Nepal. PLoS Med. 2012;9(7):e1001261.

21. Saraceno B, van Ommeren M, Batniji R, Cohen A, Gureje O, Mahoney J, et al. Barriers to improvement of mental health services in low-income and middle-income countries. Lancet. 2007:370(9593):1164-74.

22. Ventevogel P. Integration of mental health into primary healthcare in low income countries: avoiding medicalization. Int Rev Psychiatry. 2014;26(6): 669-79.

23. Eaton J, McCay L, Semrau M, Chatterjee S, Baingana F, Araya R, et al. Scale up of services for mental health in low-income and middle-income countries. Lancet. 2011:378(9802):1592-603.

24. Patel V, Bloch $\mathrm{S}$. The ethical imperative to scale up health care services for people with severe mental disorders in low and middle income countries. Postgrad Med J. 2009;85(1008):509-13.

25. Patel $V$, Maj M, Flisher AJ, De Silva MJ, KOSCHORKE M, Prince $M$, et al. Reducing the treatment gap for mental disorders: a WPA survey. World Psychiatry. 2010;9(3):169-76.

26. Semrau M, Lempp H, Keynejad R, Evans-Lacko S, Mugisha J, Raja S, et al. Service user and caregiver involvement in mental health system strengthening in low-and middle-income countries: systematic review. BMC Health Serv Res. 2016;16(1):79.

27. Lempp H, Abayneh S, Gurung D, Kola L, Abdulmalik J, Evans-Lacko S, et al. Service user and caregiver involvement in mental health system strengthening in low-and middle-income countries: a cross-country qualitative study. Epidemiol Psychiatr Sci. 2017:1-11.

28. Hanlon C, Alem A, Lund C, Hailemariam D, Assefa E, Giorgis TW, et al. Moving towards universal health coverage for mental disorders in Ethiopia. Int J Ment Heal Syst. 2019:13(1):11.

29. Ocloo J, Matthews R. From tokenism to empowerment: progressing patient and public involvement in healthcare improvement. BMJ Qual Saf. 2016; bmjqs-2015-004839.

30. Chevalier JM, Buckles DJ. Participatory action research: theory and methods for engaged inquiry: Routledge; 2013

31. Bennett M. A review of the literature on the benefits and drawbacks of participatory action research. First Peoples Child Fam Rev. 2004;14(1):109-22

32. Benjamin-Thomas TE, Corrado AM, McGrath C, Rudman DL, Hand C. Working towards the promise of participatory action research: learning from ageing research exemplars. Int J Qual Methods. 2018;17(1): 1609406918817953.

33. Rose D. Service user/survivor-led research in mental health: epistemological possibilities. Disabil Soc. 2017:32(6):773-89.

34. Näslund H, Markström U, Sjöström S. Participatory spaces of mental health service user organizations in the post-deinstitutional era: mapping roles and challenges. Volunt Int J Volunt Nonprofit Org. 2017:1-19.

35. Lindgren BM, Ringnér A, Molin J, Graneheim UH. Patients' experiences of isolation in psychiatric inpatient care: insights from a meta-ethnographic study. Int J Ment Health Nurs. 2019;28(1):7-21.

36. Leung TTF. Identity work of welfare service users in participatory spaces. Asian Soc Work Policy Rev. 2018;12(1):38-45.

37. Faulkner A. Survivor research and mad studies: the role and value of experiential knowledge in mental health research. Disabil Soc. 2017;32(4):500-20.

38. Smith E, Bélisle-Pipon J-C, Resnik D. Patients as Research Partners; How to Value their Perceptions, Contribution and Labor? Citizen Science: Theory and Practice. 2019:4(1)

39. Thornicroft G, Rose D, Mehta N. Discrimination against people with mental illness: what can psychiatrists do? Adv Psychiatr Treat. 2010;16(1):53-9.

40. Perkins A, Ridler J, Browes D, Peryer G, Notley C, Hackmann C. Experiencing mental health diagnosis: a systematic review of service user, clinician, and carer perspectives across clinical settings. Lancet Psychiatry. 2018.

41. Wallcraft J, Schrank B, Amering M. Handbook of service user involvement in mental health research: John Wiley \& Sons; 2009.

42. Jones N, Harrison J, Aguiar R, Munro L. Transforming e transformative change in mental health: towards the future. Community Psychology and 
community mental health. In: Towards transformative change; 2014. p. 35172.

43. Glasby J, Beresford P. Commentary and issues: who knows best? Evidencebased practice and the service user contribution. Crit Soc Policy. 2006;26(1): 268-84.

44. Rose D. Service user views and service user research in the journal of mental health: Taylor and Francis; 2011.

45. Russo J, editor Survivor-controlled research: a new foundation for thinking about psychiatry and mental health. Forum Qualitative Sozialforschung/ Forum: Qualitative Social Research; 2012.

46. Beresford P, Branfield F. Developing inclusive partnerships: user-defined outcomes, networking and knowledge- a case study. Health Soc Care Community. 2006;14(5):436-44.

47. Noorani T. Service user involvement, authority and the 'expert-byexperience'in mental health. J Pol Pow. 2013;6(1):49-68.

48. Boote J, Wong R, Booth A. Talking the talk or walking the walk?'a bibliometric review of the literature on public involvement in health research published between 1995 and 2009. Health Expect. 2015;18(1):44-57.

49. Brett J, Staniszewska S, Mockford C, Herron-Marx S, Hughes J, Tysall C, et al. Mapping the impact of patient and public involvement on health and social care research: a systematic review. Health Expect. 2014;17(5):637-50.

50. Henderson C, Noblett J, Parke H, Clement S, Caffrey A, Gale-Grant O, et al. Mental health-related stigma in health care and mental health-care settings. Lancet Psychiatry. 2014;1(6):467-82.

51. Thornicroft G, Mehta N, Clement S, Evans-Lacko S, Doherty M, Rose D, et al. Evidence for effective interventions to reduce mental-health-related stigma and discrimination. Lancet. 2016;387(10023):1123-32.

52. Schneider B. Participatory action research, mental health service user research, and the hearing (our) voices projects. Int J Qual Methods. 2012;11(2):152-65.

53. Rose D, Evans J, Laker C, Wykes T. Life in acute mental health settings: experiences and perceptions of service users and nurses. Epidemiol Psychiatr Sci. 2015;24(1):90-6.

54. Abma T, Banks S, Cook T, Dias S, Madsen W, Springett J, et al. Acting for Change: The Generation of Transformative Action. In: Participatory Research for Health and Social Well-Being: Springer; 2019. p. 99-124.

55. Tappan MB. Reframing internalized oppression and internalized domination: from the psychological to the sociocultural. Teach Coll Rec. 2006;108(10):2115.

56. Kemmis S, McTaggart R, Nixon R. Introducing critical participatory action research. In: The Action Research Planner: Springer; 2014. p. 1-31.

57. Bronner SE. Critical theory: A very short introduction: Oxford University press; 2017

58. Kemmis S, McTaggart R, Nixon R. Critical theory and critical participatory action research. In: The SAGE Handbook of Action Research; 2015. p. 453-44.

59. Habermas J. The theory of communicative action: Beacon press; 1984.

60. Kemmis S, McTaggart R. Participatory action research. In: Communicative Action And The Public Sphere: Sage Publications Ltd; 2005.

61. Godin P, Davies J, Heyman B, Reynolds L, Simpson A, Floyd M. Opening communicative space: a Habermasian understanding of a user-led participatory research project. J Forensic Psychiatry Psychol. 2007;18(4):452-69.

62. Wicks PG, Reason P. Initiating action research: challenges and paradoxes of opening communicative space. London: Sage Publications Sage UK; 2009.

63. Abma T, Banks S, Cook T, Dias S, Madsen W, Springett J, et al. Making the Case: The Arguments for Participatory Research. In: Participatory Research for Health and Social Well-Being: Springer; 2019. p. 1-22.

64. Habermas J. Lifeworld and system. In: A critique of functionalist reason: Beacon Press; 2003.

65. Kemmis S. Exploring the relevance of critical theory for action research: Emancipatory action research in the footsteps of Jurgen Habermas. In: Handbook of Action Research, vol. 4; 2006. p. 94-105.

66. Mill JE, Allen MN, Morrow RA. Critical theory: Critical methodology to disciplinary foundations in nursing. Can J Nurs Res Arch. 2016:33(2).

67. Kidd S, Davidson L, Frederick T, Kral MJ. Reflecting on participatory, actionoriented research methods in community psychology: Progress, problems, and paths forward. Am J Community Psychol. 2017.

68. Kincheloe JL, McLaren P. Rethinking critical theory and qualitative research In: Key Works in Critical Pedagogy: Springer; 2011. p. 285-326.

69. Mitchell PM, Roberts TE, Barton PM, Coast J. Applications of the capability approach in the health field: a literature review. Soc Indic Res. 2017;133(1): 345-71.

70. Morley C. Towards critical social work practice in mental health: a review. J Progessive Hum Serv. 2003;14(1):61-84.
71. Nelson G, Kloos B, Ornelas J. Community psychology and community mental health, Oxford University Press. In: Towards transformative change. USA; 2014.

72. Bennett $L$, Bergin M, Wells JS. The potential of critical social theory as an educational framework for people with epilepsy. Epilepsy Behav. 2016;54:80-7.

73. Reason \& Bradbury. The Sage handbook of action research: Participative inquiry and practice. Sage Publications United States of America; 2008.

74. Semrau M, Evans-Lacko S, Alem A, Ayuso-Mateos JL, Chisholm D, Gureje O, et al. Strengthening mental health systems in low-and middle-income countries: the emerald programme. BMC Med. 2015;13(1):79.

75. Emerald. Emerald: Emerging mental health systems in low- and middleincome countries. www.emerald-project.eu.

76. CSA. Summary and statistical report of the 2007 population and housing census: Federal Democratic Republic of Ethiopia Population census commission,Addis Ababa, Ethiopia 2008.

77. Hanlon C, Luitel NP, Kathree T, Murhar V, Shrivasta S, Medhin G, et al. Challenges and opportunities for implementing integrated mental health care: a district level situation analysis from five low-and middle-income countries. PLoS One. 2014;9(2):e88437.

78. Federal Ministry of Health. HSTP :Health Sector Transformation Plan- 2015/ 16-2019/20(2008-2012 EFY),Federal Democratic Republic of Ethiopia ;Ministry of Health, Addis Ababa Ethiopia.2015.

79. Wang H, Tesfaye R, Ramana GN, Chekagn CT. Ethiopia health extension program: an institutionalized community approach for universal health coverage: World Bank publications; 2016.

80. Lund C, Tomlinson M, De Silva M, Fekadu A, Shidhaye R, Jordans M, et al. PRIME: a programme to reduce the treatment gap for mental disorders in five low-and middle-income countries. PLoS Med. 2012;9(12):e1001359.

81. Fekadu A, Hanlon C, Medhin G, Alem A, Selamu M, Giorgis TW, et al. Development of a scalable mental healthcare plan for a rural district in Ethiopia. Br J Psychiatry. 2015;1:s9.

82. Fekadu A, Hanlon C, Medhin G, Alem A, Selamu M, Giorgis TW, et al. Development of a scalable mental healthcare plan for a rural district in Ethiopia. Br J Psychiatry. 2016;208(s56):s4-s12.

83. Hailemariam M, Fekadu A, Prince M, Hanlon C. Engaging and staying engaged: a phenomenological study of barriers to equitable access to mental healthcare for people with severe mental disorders in a rural African setting. Int J Equity Health. 2017;16(1):156.

84. Kemmis S, McTaggart R, Nixon R. The action research planner: doing critical participatory action research: Springer Science \& Business Media; 2013.

85. Baxter P, Jack S. Qualitative case study methodology: study design and implementation for novice researchers. Qual Rep. 2008;13(4):544-59.

86. Rouleau G, Bélisle-Pipon J-C, Birko S, Karazivan P, Fernandez N, Bilodeau K, et al. Early career researchers' perspectives and roles in patient-oriented research. Res Involvement Engagement. 2018;4(1):35.

87. Nelson G, Ochocka J, Griffin K, Lord J. "Nothing about me, without me": participatory action research with self-help/mutual aid organizations for psychiatric consumer/survivors. Am J Community Psychol. 1998;26(6): 881-912.

88. Cordeiro L, Soares CB. Action research in the healthcare field: a scoping review. JBI Database System Rev Implement Rep. 2018;16(4):1003-47.

89. Tritter JQ, McCallum A. The snakes and ladders of user involvement: moving beyond Arnstein. Health Policy. 2006;76(2):156-68.

90. Loewenson R, Laurell AC, Hogstedt C, D'Ambruoso L, Shroff Z. Participatory action research in health systems: a methods reader: regional network for equity in health in east and southern Africa (EQUINET) in association with training and research support Centre (TARSC), Alliance for Health Policy and Systems Research (AHPSR), World Health Organization (WHO) and the International Development Research Centre (IDRC) Canada; 2014.

91. Craig R-A. Empowerment and social work research-participatory action research and the relationship between the extent of mental health consumers' involvement in research and its capacity to serve an empowering function; 2008.

92. Kelly PJ. Practical suggestions for community interventions using participatory action research. Public Health Nurs. 2005;22(1):65-73.

93. Munten G, Van Den Bogaard J, Cox K, Garretsen H, Bongers I. Implementation of evidence-based practice in nursing using action research: a review. Worldviews Evid Based Nurs. 2010;7(3):135-57.

94. Roberts L. Community-based participatory research for improved mental healthcare: A manual for clinicians and researchers: Springer Science \& Business Media; 2012. 
95. Ghaye T, Melander-Wikman A, Kisare M, Chambers P, Bergmark U, Kostenius $C$, et al. Participatory and appreciative action and reflection (PAAR)democratizing reflective practices. Reflective Pract. 2008;9(4):361-97.

96. MacDonald C. Understanding participatory action research: a qualitative research methodology option. Can J Action Res. 2012;13(2):34-50.

97. Waterman H, Tillen D, Dickson R, De Koning K. Action research: a systematic review and guidance for assessment. Health Technol Assess(Winchester, England). 2001;5(23):iii.

98. Chen HT. Practical program evaluation: theory-driven evaluation and the integrated evaluation perspective: Sage Publications; 2014.

99. Rogers PJ. Using programme theory to evaluate complicated and complex aspects of interventions. Evaluation. 2008;14(1):29-48.

100. Apgar JM, Allen W, Albert J, Douthwaite B, Paz Ybarnegaray R, Lunda J. Getting beneath the surface in program planning, monitoring and evaluation: learning from use of participatory action research and theory of change in the CGIAR research program on aquatic agricultural systems. Action Res. 2017;15(1):15-34.

101. Douthwaite B, Hoffecker E. Towards a complexity-aware theory of change for participatory research programs working within agricultural innovation systems. Agric Syst. 2017;155:88-102.

102. Janzen R, Ochocka J, Stobbe A. Towards a theory of change for communitybased research projects. Engaged Scholar J Community-Engaged Res Teach Learn. 2016;2(2):44-64.

103. Whynot J, Lemire S, Montague S. How We Model Matters: A Manifesto for the Next Generation of Program Theorizing. Can J Program Eval. 2019;33(3):414-33.

104. Koleros A, Mayne J. Using Actor-Based Theories Of Change to Conduct Robust Contribution Analysis in Complex Settings. Can J Program Eval. 2019;33(3):292-315.

105. Montague S. Does Your Implementation Fit Your Theory of Change? Can J Program Eval. 2019;33(3):316-35.

106. Rogers PJ. Implications of methodological and procedural choices in equityfocused evaluations. Eval Equitable Soc. 2016;199.

107. Montague S, Porteous NL. The case for including reach as a key element of program theory. Eval Program Plann. 2013;36(1):177-83.

108. Avila EM, Tolentino LL, Binondo CB, Perez ML, Apgar JM. Building bridges to an uncertain future lived now: lessons from the use of participatory action research and theory of change towards a realistic community-based participatory monitoring and evaluation system. Int J Agric Syst. 2016;4(1): 85-106.

109. Archibald T, Sharrock G, Buckley J, Cook N. Assumptions, conjectures, and other miracles: the application of evaluative thinking to theory of change models in community development. Eval Program Plann. 2016;59:119-27.

110. de Brun T, O'Reilly-de Brun M, O'Donnell CA, MacFarlane A. Learning from doing: the case for combining normalisation process theory and participatory learning and action research methodology for primary healthcare implementation research. BMC Health Serv Res. 2016;16(a):346.

111. Chen $\mathrm{H}, \mathrm{H}-\mathrm{LW}$ P, Morosanu L, Turner N. Using logic models and the action model/change model schema in planning the learning community program: A comparative case study. Can J Program Eval. 2018;33(1):49-68.

112. Casey M, O'Leary D, Coghlan D. Unpacking action research and implementation science: implications for nursing. J Adv Nurs. 2018;74(5):1051-8.

113. Sprague L, Afifi R, Ayala G, El-nasoor ML. Participatory praxis as an imperative for health-related stigma research. BMC Med. 2019;17(1):32

114. Sullivan H, Barnes M, Matka E. Building collaborative capacity throughTheories of Change' early lessons from the evaluation of health action zones in England. Evaluation. 2002;8(2):205-26.

115. Hart D. A systemic 'Theories of Change'approach for projects and change initiatives in the context of quality enhancement activity in Higher Education. In: Problem Structuring Approaches for the Management of Projects: Springer; 2019. p. 1-34.

116. Guarneros-Meza V, Downe J, Martin S. Defining, achieving, and evaluating collaborative outcomes: a theory of change approach. Public Manag Rev. 2018;20(10):1562-80

117. Barnes M, Matka E, Sullivan H. Evidence, understanding and complexity: evaluation in non-linear systems. Evaluation. 2003;9(3):265-84.

118. Bolton N, Martin S, Grace C, Harris S. Implementing a theory of change approach to research sport participation programmes targeting 'hard to reach'groups. Int J Sport Policy and Polit. 2018:1-17.

119. Dickinson $H$, Sullivan $H$. Towards a general theory of collaborative performance: the importance of efficacy and agency. Public Adm. 2014;92(1):161-77.
120. Ward M, Schulz AJ, Israel BA, Martenies SE, Markarian E. A Conceptual Framework for Evaluating Health Equity Promotion within CommunityBased Participatory Research Partnerships. Eval Program Plann. 2018; 70(2018):25-34

121. Brydon-Miller M, Rector Aranda A, Stevens DM. Widening the circle: ethical reflection in action research and the practice of structured ethical reflection. In: The SAGE handbook of action research; 2015. p. 596-607.

122. Lund R, Panda SM, Dhal MP. Narrating spaces of inclusion and exclusion in research collaboration-researcher-gatekeeper dialogue. Qual Res. 2016;16(3):280-92.

123. Hall AE, Bryant J, Sanson-Fisher RW, Fradgley EA, Proietto AM, Roos I. Consumer input into health care: time for a new active and comprehensive model of consumer involvement. Health Expect. 2018;21(4):707-13.

124. Smith L, Bratini L, Chambers D-A, Jensen RV, Romero L. Between idealism and reality: meeting the challenges of participatory action research. Action Res. 2010:8(4):407-25.

125. Hewstone M, Swart H. Fifty-odd years of inter-group contact: from hypothesis to integrated theory. Br J Soc Psychol. 2011;50(3):374-86.

126. Frías VM, Fortuny JR, Guzmán S, Santamaría P, Martínez M, Perez V. Stigma: the relevance of social contact in mental disorder. Enfermería Clínica (English Edition). 2017;28(2):111-17.

127. Pattyn E, Verhaeghe M, Bracke P. Attitudes toward community mental health care: the contact paradox revisited. Community Ment Health J. 2013; 49(3):292-302

128. Cerully JL, Collins RL, Wong E, Seelam R, Yu J. Differential response to contact-based stigma reduction programs: perceived quality and personal experience matter. Psychiatry Res. 2018;259:302-9.

129. Kemmis S, McTaggart R. The Action Research Planner: Deakin University; 1988.

130. White GW, Suchowierska M, Campbell M. Developing and systematically implementing participatory action research. Arch Phys Med Rehabil. 2004; 85:3-12.

131. Mackenzie M, Blamey A. The practice and the theory: lessons from the application of a theories of change approach. Evaluation. 2005;11(2):151-68.

132. Prinsen G, Nijhof S. Between logframes and theory of change: reviewing debates and a practical experience. Dev Pract. 2015;25(2):234-46.

133. Weiss $\mathrm{CH}$. Theory-based evaluation: past, present, and future. N Dir Eval. 1997;1997(76):41-55

134. Cahill C, Torre ME. Beyond the journal article: Representations, audience, and the presentation of participatory action research. Routledge: Participatory action research approaches and methods; 2007. p. 222-31.

135. Cowan K, Guidebook OSJLA. James Lind Alliance Gudebook (version 5). Southampton: James Lind Alliance; 2013.

136. Evans N, Hannigan B, Pryjmachuk S, Gillen E, Edwards D, Longo M, et al. Using the nominal group technique to involve young people in an evidence synthesis which explored 'risk'in inpatient mental healthcare. Res Involvement Engagement. 2017;3(1):16.

137. Thier M, Mason DP. Breaking ranks? Differentiating nominal group technique scoring approaches for consensus and prioritization. Int J Res Method Educ. 2018:42(issue 4):1-14.

138. Harvey N, Holmes CA. Nominal group technique: an effective method for obtaining group consensus. Int J Nurs Pract. 2012:18(2):188-94.

139. Hollis C, Sampson S, Simons L, Davies EB, Churchill R, Betton V, et al. Identifying research priorities for digital technology in mental health care: results of the James Lind Alliance priority setting partnership. Lancet Psychiatry. 2018;5(10):845-54

140. Abayneh S, Lempp H, Alem A, Alemayehu D, Eshetu T, Lund C, et al. Service user involvement in mental health system strengthening in a rural African setting: qualitative study. BMC Psychiatry. 2017;17(1):187.

141. Ochocka J, Moorlag E, Janzen R. A framework for entry: PAR values and engagement strategies in community research. Gateways Int J Community Res Engagement. 2010;3:1-19.

142. Braun V, Clarke V. Successful qualitative research: A practical guide for beginners: sage; 2013.

143. Pietkiewicz I, Smith JA. A practical guide to using interpretative phenomenological analysis in qualitative research psychology. Psychol J. 2014;20(1):7-14.

144. Stringer ET. Action research: sage publications; 2013.

145. Guba EG, Lincoln YS. Paradigmatic controversies, contradictions, and emerging confluences; 2005.

\section{Publisher's Note}

Springer Nature remains neutral with regard to jurisdictional claims in published maps and institutional affiliations. 